\title{
HIV medicine: drug side effects and interactions
}

\author{
Christine M Bates
}

\begin{abstract}
Summary
The drugs used in HIV medicine often have toxic side effects; additionally, the risk of drug interactions is high because of the frequent necessity to prescribe multiple drugs. This article covers common or important drug side effects and interactions.
\end{abstract}

Keywords: HIV, AIDS, drug side effects, drug interactions

\section{Case report}

A 44-year-old man was admitted with pneumonia which had been unresponsive to conventional broad spectrum antibiotics. Chest X-ray appearances were suggestive of PCP which was confirmed by bronchial lavage. Tests for HIV antibodies were positive. Treatment was changed to intravenous cotrimoxazole $120 \mathrm{mg} / \mathrm{kg}$ daily; six days later a widespread, maculopapular, pruritic rash developed. Cotrimoxazole was discontinued and nebulised pentamidine substituted. The rash resolved within a week

\section{Box 1}

\begin{tabular}{|l|}
\hline Cotrimoxazole: side effects \\
\hline - maculopapular rash \\
- Stevens-Johnson syndrome \\
- toxic epidermal necrolysis \\
- nausea, vomiting and diarrhoea \\
- pseudomembranous colitis \\
- pancreatitis \\
- raised liver function tests \\
- haepatic necrosis \\
eosinophological disorders: \\
granulocytopenia, leucopenia, \\
thrombocytopenia, megaloblastic \\
anaemia
\end{tabular}

Box 2

AIDS Clinical Group, Royal Liverpool
University Hospital, Liverpool L7 8XP,
UK
CM Bates

CM Bates
Patients infected with HIV display an increased incidence of hypersensitivity reactions which often necessitate a change in therapy. The pathogenesis of these reactions is unclear. However, possible factors include the production of toxic metabolites combined with genetic and acquired alterations in metabolic capacity and acquired deficiency in glutathione. ${ }^{1}$

\section{Drugs used to treat Pneumocystis carinii pneumonia}

COTRIMOXAZOLE (trimethoprim-sulphamethoxazole)

It is well recognised that adverse drug reactions to cotrimoxazole occur commonly in HIV-positive patients. ${ }^{2,3}$ The overall number of reactions is greatly increased compared with the general population (up to $100 \%{ }^{4}$ compared with $2-8 \%{ }^{5}$ ) although the range of reactions is similar. The commonest observed reaction is a generalised maculopapular rash, which may be pruritic, occurring one to two weeks after starting treatment (box 1).

A study comparing trimethoprim-sulphamethoxazole with trimethoprim-dapsone found that although both treatments were equally effective in the treatment of mild/moderate first episodes of Pneumocystis carinii pneumonia (PCP), twice as many major events were recorded in those receiving cotrimoxazole. ${ }^{6}$ The incidence of chemical hepatitis (aspartate transaminase, alanine transaminase and/or alkaline phosphatase increased to five times normal levels) and neutropenia $\left(<750\right.$ neutrophils $/ \mathrm{mm}^{3}$ ) was very much more common in the cotrimoxazole group, although rash and fever occurred with equal frequency in both groups. Other reactions to cotrimoxazole are summarised in box 2 .

Cotrimoxazole increases the antifolate effects of methotrexate, pyrimethamine and phenytoin (plasma concentration is also increased.) The effects of sulphonylureas, nicoumalone and warfarin are also enhanced.

\section{DAPSONE}

Dapsone is used with trimethoprim as an alternative regime for PCP. As mentioned above, the incidence of adverse reactions with this combination compared to cotrimoxazole is lower, $(30 \%$ vs $57 \%$ ). Methaemoglobinaemia is found in most HIV-positive patients taking dapsone but is usually asymptomatic. Mild hyperkalaemia ( $\mathrm{K}>5-6.1 \mathrm{mmol} / 1)$ has been reported to occur in up to $53 \%$ of patients. ${ }^{6}$ Other (dose-related) effects include neuropathy, allergic dermatitis, anorexia, nausea, vomiting, headache, insomnia, tachycardia, anaemia, hepatitis and agranulocytosis.

Dapsone and trimethoprim used in combination increases the plasma concentrations of both drugs. ${ }^{7}$ Rifampicin, as a result of enzyme induction, may decrease the half-life of dapsone while probenicid may reduce its urinary excretion.

\section{PENTAMIDINE}

Pentamidine is used in the treatment and prophylaxis of PCP and may be given intravenously or by nebulisation. Major adverse effects are summarised in box 3 . Other reactions are listed in box 4. A comparison of pentamidine with cotrimoxazole for PCP showed that the rate of minor adverse reactions was the same in both groups. ${ }^{4}$ Bronchoconstriction occurring with nebulised pentamidine may be prevented or ameliorated by prior inhalation of a bronchodilator.

Pancreatitis has been reported in association with both intravenous and aerosolised pentamidine. ${ }^{8,9}$

\section{CLINDAMYCIN}

Nausea and diarrhoea are the commonest side effects; the latter, more frequently seen with oral than with parenteral treatment, is an indication to stop therapy. Pseudomembranous colitis may also develop. A generalised maculopapular rash with clindamycin, both where it has been used in conjunction with primaquine in 


\begin{tabular}{|l|}
\hline Pentamidine: major \\
adverse effects \\
\hline - hypotension \\
- hypoglycaemia \\
- pancreatitis \\
- arrhythmias \\
\hline
\end{tabular}

Box 3

\begin{tabular}{|l|}
\hline Pentamidine: side effects \\
\hline - leucopenia \\
- thrombocytopenia \\
- anaemia \\
- hytaemia \\
- acute renal failure \\
- hypocalcaemia \\
- hyperglycaemia \\
- abnormal liver function tests \\
- nausea and vomiting \\
- synziness \\
- flushing \\
- rash \\
- taste disturbances \\
- local reaction at injection site \\
\hline
\end{tabular}

Box 4 the treatment of PCP and also where it has been used singly as in toxoplasmosis, has been observed.

Elevated serum transaminases, reversible neutropenia, eosinophilia, agranulocytosis and thrombocytopenia have occasionally been reported, although it appears that clindamycin may be given concurrently with zidovudine without adding to its myelotoxic effects.

Concurrent administration of erythromycin causes displacement of clindamycin from its ribosomal binding site and should be avoided.

\section{PRIMAQUINE}

Primaquine, which is used in conjunction with clindamycin in the treatment of PCP, may cause nausea, vomiting and abdominal pain. Methaemoglobinaemia is an uncommon side effect as is haemolytic anaemia which is more severe in patients with glucose-6-phosphate dehydrogenase deficiency.

\section{ATOVAQUONE}

The most common adverse experiences reported are rash, nausea, diarrhoea, headache, vomiting, fever and insomnia. Raised liver function tests and amylase, hyponatraemia and anaemia have also been noted. Bioavailability is increased two- to four-fold when atovaquone is taken with food, particularly high fat food. The presence of diarrhoea at the start of treatment is associated with lower plasma levels. Concurrent treatment with rifampicin or metoclopramide leads to significantly reduced plasma concentrations of atovaquone. The rate of glucuronidation of zidovudine is decreased by atovaquone; adverse reactions are more likely to occur in patients taking high doses of zidovudine or those receiving a prolonged course of atovaquone.

\section{Drugs used to treat mycobacterial infections}

\section{ANTITUBERCULOUS TREATMENT}

Adverse drug reactions occur more frequently in patients with HIV infection who are receiving antituberculous medications (including rifampicin, thiacetazone (not used in UK), isoniazid, ethambutol, pyrazinamide and streptomycin) than in comparable HIV-negative groups. ${ }^{10}$ In combination therapy it is not always possible to tell which drug has caused a reaction but rifampicin appears to be the most commonly implicated out of this group.

\section{Rifampicin}

Side effects of rifampicin include gastrointestinal symptoms and disorders of liver function including jaundice. An influenza-like syndrome (fever, dizziness and bone pain), respiratory symptoms, collapse and shock, haemolytic anaemia, acute renal failure or thrombocytopenic purpura mainly occur on intermittent treatment. Rifampicin causes orange-red discolouration of body fluids such as urine, tears, sweat and saliva. Anaphylactoid reactions have been reported. ${ }^{11}$ Most major side effects occur within the first two months of treatment. ${ }^{12}$

Drug interactions include the following: reduced blood levels of ketoconazole, fluconazole ${ }^{13}$ and itraconazole caused by enzyme induction. Half-lives of trimethoprim and tolbutamide are decreased. Serum levels of dapsone, methadone, oral contraceptives, corticosteroids, coumarin anticoagulants and theophylline are reduced.

\section{Rifabutin}

See under Drugs used to treat Mycobacterium avium intracellulare below.

\section{Isoniazid}

The commonest side effect is a peripheral neuropathy which is more frequently seen in slow acetylators and in patients with risk factors such as malnutrition, diabetes, alcoholism or chronic renal failure. It is responsive to pyridoxine which should, therefore, be given prophylactically if any of these factors is known to exist.

A rise in serum transaminases is often seen; this is more common in older patients, fast acetylators, patients with a high intake of alcohol or pre-existing liver disease. Hepatitis is rare, as is psychosis. Other side effects include nausea and rash.

Drug interactions include a decrease in absorption of isoniazid if given with aluminium-containing antacids, and raised serum levels of phenytoin, carbamazepine, warfarin, benzodiazepines and theophylline due to inhibition of metabolism. 
Ethambutol

The main side effects of ethambutol are of ocular toxicity, the symptoms of which include blurred vision, colour blindness and restriction of visual fields. These effects are dose related or seen in patients whose renal function is impaired and are almost always reversible if the drug is discontinued early.

\section{Pyrazinamide}

Hepatotoxicity is the most important, although not common, side effect of pyrazinamide; it is associated with high, prolonged doses and may present with fever, hepatomegaly, jaundice, liver failure and anorexia. Arthralgia, nausea and vomiting are also side effects as are raised serum levels of uric acid.

Pyrazinamide given concurrently with probenicid leads to an increased half-life of probenicid which counteracts the suppression of urate excretion due to pyrazinamide.

\section{DRUGS USED TO TREAT MYCOBACTERIUM AVIUM INTRACELLARE \\ Rifabutin}

A member of the rifamycin group, rifabutin, like rifampicin, may cause red-orange discolouration of skin, urine and other body secretions. Adverse effects include nausea, vomiting, jaundice, raised liver function tests, arthralgia, myalgia, and haematologic reactions. Rash, fever and anaphylaxis have also been reported. Reversible uveitis, which may be severe, has occurred where rifabutin is used at higher doses, especially when combined with clarithromycin (or other macrolides). Rifabutin induces the enzymes of cytochrome P450 3A subfamily and, therefore, may be expected to affect plasma concentrations of drugs metabolised by these enzymes, for example, oral contraceptives, analgesics, corticosteroids, dapsone and phenytoin. Plasma levels of rifabutin are increased by concomitant clarithromycin and/or fluconazole.

\section{Ciprofloxacin}

In common with other members of the 4-quinolone group of antibacterials, ciprofloxacin may be associated with a wide range of side effects. Nausea, vomiting, abdominal pain and diarrhoea may occur in up to $10 \%$ of patients. Other side effects include headache, dizziness, rash, pruritis, disturbances of renal and hepatic function and haematological disorders. Convulsions may occur with or without a history of epilepsy, the risk is increased if nonsteroidal anti-inflammatory drugs are taken concurrently. Anaphylaxis has been reported $^{11}$; it is more common in HIV-positive patients and can be fatal. ${ }^{14}$

Antacids, sulcrafate and oral iron reduce the absorption of ciprofloxacin. Theophylline plasma concentration and the anticoagulant effect of warfarin and nicoumalone are increased by concurrent administration of ciprofloxacin.

\section{Clofazamine}

Nausea, giddiness, headache and diarrhoea are dose-related side effects. Patients should be warned that a reversible red discolouration of skin and urine may occur. Visual acuity may be adversely affected during long-term treatment due to retinal degeneration.

\section{Cycloserine}

The main side effects are neurological: headache, dizziness, vertigo, drowsiness, tremor and convulsions. Psychosis, depression and rash have been reported. Monitoring of haematological, renal and hepatic function is necessary as megaloblastic anaemia and changes in liver function may occur; renal impairment increases the likelihood of toxicity.

\section{Drugs used to treat fungal infections}

\section{FLUCONAZOLE}

Generally well tolerated, this drug may cause nausea, headache, rash and raised liver function tests. It is less $\mathrm{pH}$-dependent than ketoconazole for absorption.

Important drug interactions include the reduction of the half-life of fluconazole by rifampicin. ${ }^{13}$ Fluconazole given concurrently increases levels of phenytoin, sulphonylureas (increased risk of hypoglycaemia), and rifabutin (leading to a risk of uveitis). Fluconazole decreases the metabolism of warfarin, thus increasing its anticoagulant effect.

Nausea and vomiting occur as common side effects; rashes and, rarely, thrombocytopenia, gynaecomastia and fatal liver damage have been reported. Bioavailability is reduced in HIV-positive patients due to gastric hypochlor- 
hydria. Levels are reduced where there is concurrent administration of $\mathrm{H} 2$-antagonists or antacids. This interaction can be overcome by delaying the ingestion of antacid by at least two hours after that of ketoconazole or by giving the ketoconazole with an acidic drink such as fruit juice. Rifampicin should not be given with ketoconazole as a substantial reduction in the serum level of the latter occurs. Metabolism of astemizole and terfenadine is inhibited, leading to possible cardiac toxicity.

\section{ITRACONAZOLE}

Usually well tolerated, itraconazole may cause nausea, dyspepsia, headache and abdominal pain. Elevation of liver function tests, allergic reactions and StevensJohnson syndrome have been reported. Absorption is improved if it is taken with food and, in common with ketoconazole, problems with absorption may arise due to gastric achlorhydria or interactions with $\mathrm{H} 2$-antagonists and antacids. Serum levels of itraconazole are reduced when phenytoin or rifampicin are given concurrently. Itraconazole increases the effects of anticoagulants, midazolam, triazolam and should not be given with astemizole or terfenadine because of the risk of cardiac toxicity.

\section{FLUCYTOSINE}

Diarrhoea, nausea, vomiting and rash occur as side effects of this drug. Abnormal liver function tests may be found in $5 \%$ of patients; liver necrosis has been reported. Dose-related toxic effects include agranulocytosis, aplastic anaemia and thrombocytopenia. Regular monitoring of blood counts during prolonged therapy is therefore recommended. Where patients are also taking other bone marrow toxic medications the effects may be additive. Synergy occurs with amphotericin. Anaphylactoid reaction has been reported in an HIV-positive patient. $^{15}$

\section{AMPHOTERICIN B}

Amphotericin B may cause renal impairment, necessitating a change in treatment. Other side effects include rash, febrile reactions, anaphylactoid reactions, elevated liver function tests and electrolyte abnormalities, such as hypokalaemia and hypomagnesaemia. Other side effects include anorexia, nausea, vomiting, diarrhoea, headache, myalgia and arthralgia. Toxic cardiovascular and neurological effects may occur.

Concurrent administration of digitalis, carbenicillin or ticarcillin may exacerbate the hypokalaemic effect. Liposomal amphotericin B causes significantly less renal toxicity, although recently anaphylactoid reactions have been reported ${ }^{16}$ (box 5).

A 28-year-old man, HIV positive for nine years, had received conventional intravenous amphotericin B twice over the proceeding nine months as treatment for cryptococcal meningitis. On the second occasion renal impairment had developed and fluconazole was substituted. Despite maintenance treatment, with fluconazole initially and itraconazole subsequently, the infection recurred three months later and therefore liposomal amphotericin B was given. Within seconds of commencing his first injection he vomited and complained of epigastric pain. Bronchospasm, facial flushing and sweating were noted. The infusion was immediately discontinued and symptoms settled within four hours

\section{Box 5}

\begin{tabular}{|l|}
\hline Zidovudine: haematological \\
effects \\
\hline - anaemia \\
- neutropenia \\
- leucopenia \\
- thrombocytopenia \\
- pancytopenia \\
\hline
\end{tabular}

\section{Antiretroviral drugs}

\section{ZIDOVUDINE}

The haematological effects (box 6) are directly related to dosage and treatment duration of zidovudine. Significant anaemia, which may require transfusion, develops between four and six weeks after starting therapy. These toxic effects are inversely related to the haemoglobin concentration, granulocyte and CD4 lymphocyte counts. Other side effects include fever, rash, and gastrointestinal symptoms. Fatty liver change may occur and hepatomegaly associated with elevated liver function tests is an indication to stop treatment as is the development of lactic acidosis. Long-term treatment may result in myopathy, associated with an elevated creatinine kinase and characterised by proximal muscle wasting, in up to $20 \%$ of patients; nail hyperpigmentation is also a consequence of prolonged therapy.

Probenicid increases the half-life of zidovudine by inhibiting its renal tubular secretion..$^{17}$ The dosage of zidovudine should therefore be adjusted to avoid the risk of increased toxicity; by the same token probenicid may be given so that the daily dosage of zidovudine can be reduced. Clarithromycin reduces the plasma concentration of zidovudine, probably by inhibiting absorption, but this can be avoided by administering the two drugs with a time interval of one or two hours between doses. ${ }^{18}$

\section{2', 3' - DIDEOXYINOSINE (ddI)}

Pancreatitis and peripheral neuropathy occur as toxic effects of ddI. Patients with a history of pancreatitis, a low CD4 count $\left(<50 / \mathrm{mm}^{3}\right)$ and advanced HIV disease are more likely to develop pancreatitis. The reversible neuropathy is related to dosage and duration of treatment. Other side effects include deranged liver function tests, hyperuricaemia (related to high dose), abdominal pain and 
abnormalities in cardiac function. Optic neuritis, fulminant hepatitis and Stevens-Johnson syndrome ${ }^{19}$ have also been described.

As ddI is prepared with an alkaline buffer, absorption of drugs which need an acidic environment, such as dapsone and ketoconazole, is reduced and therefore these drugs should be given at least two hours before ddI. Absorption of quinolone antibiotics and tetracycline may also be reduced by concurrent ddI.

\section{ZALCITABINE (ddC)}

Peripheral neuropathy is a serious adverse side effect which may be irreversible unless treatment is stopped immediately. The development of pancreatitis, lactic acidosis, fatty liver change or hepatomegaly are all also indications for immediate cessation of ddC therapy. Other side effects include mucositis, rash, fever, rigors, fatigue, weight loss, dizziness, headache, pruritis, sweating, chest pain, gastrointestinal symptoms, myalgia, arthralgia, haematological symptoms (anaemia, neutropenia, leucopenia and thrombocytopenia) and abnormal liver function tests.

\section{Drugs used to treat cytomegalovirus infection}

\section{GANCICLOVIR}

Neutropenia, the most severe adverse drug reaction, is dose related and reversible, occurring in up to $42 \%$ of patients. ${ }^{20} \mathrm{GM}-\mathrm{CSF}$, which is well tolerated and biologically active in patients with AIDS, may be used to correct or prevent this drug-induced neutropenia and thus allow continuation of treatment. ${ }^{21}$ Twenty per cent of patients are affected by thrombocytopenia, which is more likely to develop where the pre-existing count is $<100 \times 10^{9} / 1$. Anaemia, fever, rash, and elevation of liver function tests may also occur.

Zidovudine should not be given concurrently during high-dose induction therapy with ganciclovir because of the additive neutropenic effects; severe neutropenia may also result if zidovudine is given during maintenance treatment. Probenicid increases the half-life of ganciclovir; additive toxicity may be seen with dapsone, pentamidine, flucytosine, amphotericin B, cotrimoxazole, vincristine, vinblastine and adriamycin.

\section{FOSCARNET}

Side effects include nausea, vomiting, headache, fatigue and rash. Impairment of renal function (including acute renal failure), and symptomatic hypocalcaemia (increased risk with concurrent pentamidine), have been reported. Anaemia, hypoglycaemia and convulsions have occasionally occurred. Painful penile ulcers negative for cytomegalovirus, herpes simplex virus and treponemes may develop 5-24 days after starting treatment (high-dose induction). Resolution may occur on maintenance doses or up to 10 days after stopping treatment. ${ }^{22}$ In some patients similar oral lesions have developed. ${ }^{23}$

\section{Drugs used to treat herpes simplex and varicella zoster}

\section{ACYCLOVIR}

Generally well tolerated the commonest side effects on oral therapy are nausea, (seen in up to $8 \%$ of patients,) and headache, (occurring in up to $6 \%$ of patients.) Rashes, fatigue, elevated liver function tests, urea and creatinine have been noted. A reversible nephropathy due to crystalline precipitation in the renal tubules may occur where dehydration, pre-existing renal insufficiency and high-dose bolus infusion are predisposing factors. Intravenous acyclovir has been associated with neuropsychiatric reactions.

Probenicid reduces the excretion of acyclovir thus leading to raised plasma concentrations. Zidovudine given concurrently with intravenous acyclovir may cause extreme lethargy.

\section{Drugs used to treat toxoplasmosis}

\section{CLINDAMYCIN}

See under Drugs used to treat PCP above.

\section{PYRIMETHAMINE}

Pyrimethamine and sulfadiazine are used together to treat toxoplasmosis; toxic reactions are common and include rash, bone marrow toxicity and raised liver enzyme levels. Stevens-Johnson syndrome may occur due to the sulphonamide component. The bone marrow effects are potentiated by other medications with similar activity such as zidovudine. Additionally, where pyrimethamine and 
cotrimoxazole are given together, pancytopenia and megaloblastic anaemia may occur because of the adverse effect on folate metabolism.

\section{Drugs used in Kaposi's sarcoma}

Discussion of the drugs used in the chemotherapy of Kaposi's sarcoma is outside the scope of this article. However, it is important to note that the neutropenic effects of medications such as bleomycin, vincristine and etoposide may be exacerbated by concomitant use of drugs such as ganciclovir, zidovudine, dapsone and cotrimoxazole, the latter two by means of their effect on folate metabolism.

\section{Anticonvulsants}

There have been cases reported of fever associated with erythematous maculopapular eruptions in HIV-positive patients receiving carbamazepine or phenytoin; these reactions occurred during the second week of treatment in each case. Additionally, phenytoin has been associated with Stevens-Johnson syndrome and toxic epidermal necrolysis, as have valproate, chloral hydrate and lorazepam.

\section{Amoxycillin}

A 26-year-old, pregnant, HIV-positive, Zimbabwean woman developed a submandibular abscess due to Mycobacterium fortuitum. During the course of treatment she developed nausea and vomiting with clarithromycin and a rash due to amikacin. Prophylaxis for PCP was given with cotrimoxazole initially, but after a widespread itchy rash developed, this was changed to dapsone. After delivery, cefoxitin, co-amoxyclav and doxycycline were used in treatment of the abscess but co-amoxyclav caused itching and cefoxitin infusion caused chest symptoms including cough which resolved after the infusion was stopped. Doxycycline ingestion induced nausea, vomiting and chest discomfort but this was overcome by changing to a dispersible preparation. Neutropenia developed on dapsone and PCP prophylaxis was changed to nebulised pentamidine

Box 7

\section{Summary/learning points}

- individuals with HIV/AIDS are more likely to have drug side-effects

- such individuals are often on several medications, which might interact

Box 8

1 Koopmans PP, van der Ven AJAM, Vree TM, van der Meer JWM. Pathogenesis of hypersensitivity reactions to drugs in patients with HIV infection: allergic or toxic? AIDS 1995; 9, 217-22.

2 Gordin FM, Simon GL, Wofsy CB, Mills J Adverse reactions to trimethoprim-sulfamethoxazole in patients with the acquired immunodeficiency syndrome. Ann Intern Med 1984; 100: 495-9.

3 Jaffe HS, Abrahams D, Ammon AJ, Lewis BJ, Golden JA. Complications of cotrimoxazole in treatment of AIDS associated Pneumocystis carinii pneumonia in homosexual men. Lancet carinii pneumonia in

Erythematous, pruritic, macular rashes mainly affecting the limbs and trunk (rarely the face) are common in HIV-positive patients when treated with amoxycillin-clavulanate. ${ }^{24}$ The rash may occur 3-21 days after commencing treatment and is more frequent in patients with a low CD4 count $(<200 / \mathrm{ml})$.

Other side effects include nausea, diarrhoea and, rarely, pseudomembranous colitis.

\section{Thalidomide}

Peripheral neuropathy and teratogenicity are the main side effects of thalidomide. Others include rash and anaphylactoid reactions. Thalidomide has been used to treat intractable oropharyngeal aphthous ulceration in patients with Stage IV HIV disease. ${ }^{25}$ In this study seven out of eight patients experienced pain relief and healing. However, three developed a widespread erythematous macular rash after 6-12 days of treatment.

\section{Multiple drugs}

As mentioned above, the occurrence of multiple drug reactions in HIV-positive patients is greater than in the general population. Wijnants and colleagues describe an HIV-positive patient with a history of rash with cotrimoxazole and amoxycillin. ${ }^{26} \mathrm{He}$ was subsequently found to develop rash and Stevens-Johnson syndrome during the course of treatment with ethambutol, isoniazid, rifampicin and ofloxacin. Symptoms resolved after stopping treatment but he required treatment with steroids for a rash and fever which occurred after meglumine ioxitalamine was injected intravenously during a CT brain scan. A review of 33 AIDS patients admitted consecutively showed that adverse drug reactions occurred in $12 \%$. Other case reports of multiple drug reactions include the patient described in box 7 and a patient who had rash and fever with 10 different medications. ${ }^{27}$

I am grateful to Dr P B Carey and Dr C R K Hind for giving permission to use their patients as case reports.

4 Warton JM, Coleman DL, Wofsy CB, et al. Trimethoprim-sulfamethoxazole or pentamidine for Pneumocystis carinii pneumonia in the acquired immunodeficiency syndrome: a proacquired immunodeficiency syndrome: a prospective random $37-44$.

105: $37-44$.
5 Jick J. Adverse reactions to trimethoprimsulfamethoxazole in hospitalized patients. $R e v$ Infect Dis 1982; 4: 426-8.

6 Medina I, Mills J, Leoung G, et al. Oral therapy for Pneumocystis carinii pneumonia in the Acquired Immunodeficiency Syndrome: a contAcquired Immunodeficiency Syndrome: a cont-
rolled trial of trimethoprim-sulfamethoxazole rolled trial of trimethoprim-sulfamethoxazole
versus trimethoprim-dapsone. $N$ Engl $₹ \mathrm{Med}$ 1990; 323: 776-82.
7 Lee BL, Medina I, Bernowitz NL, Jacob P Wofsy CB, Mills J. Dapsone, trimethoprim and sulfamethoxazole plasma levels during treatment of Pneumocystis pneumonia in patients with acquired immunodeficiency syndrome: evidence of drug interactions. Ann Intern Med 1989; 110: 606-11.

8 O'Neill MG, Selub SE, Hak LJ. Pancreatitis during pentamidine therapy in patients with AIDS. Clin Pharm 1991; 10: 56-8.

9 Singh G, El-Gadi SM, Sparks RA. Pancreatitis associated with aerosolised pentamidine Genitourin Med 1995; 71: 130-1 
10 Pozniak AL, Macleod GA, Maxwell M, Legg W, Weinberg $J$. The influence of HIV status on single and multiple drug reactions to antituberculous therapy in Africa. AIDS 1992; 6: 809-14.

11 Wurtz RM, Abrams D, Becker S, Jacobson MA Mass MM, Marks SH. Anaphylactoid drug reactions to ciprofloxacin and rifampicin in HIV-infected patients. Lancet 1989; 1: 955-6.

12 Small PM, Schecter GF, Goodman PC, Sande MA, Chaisson RE, Hopewell PC. Treatment of tuberculosis in patients with advanced human immunodeficiency virus infection. $N \mathrm{Engl} f$ Med 1991; 324: 289-94.

13 Coker RJ, Tomlinson DR, Parkin J, Harris JR, Pinching AJ. Interaction between fluconazole and rifampicin. $B M F$ 1990; 301: 818 .

14 Peters J. Fatal anaphylaxis to ciprofloxacin in an AIDS patient. $B M \mathcal{F}$ 1989; 298: 605.

15 Kotani S, Hirose S, Niiya K, Kubonishi I, Miyoshi I. Anaphylaxis to flucytosine in a Miyoshi 1. Anaphylaxis to flucytosine in a

patient with AIDS. $\mathcal{F} A M A$ 1988; 260: $3275-6$.
16 Laing RBS, Milne LJR, Leen CLS, Malcolm GP, Steers AJW. Anaphylactic reactions to liposomal amphotericin. Lancet 1994; 344: 682.
17 Kornhauser DM, Petty BG, Hendrix CW, et al. Probenicid and zidovudine metabolism. Lance 1989; ii: 473-5.

18 Gustavson LE, Chu SY, Mackenthun A, Gupta $\mathrm{SD}$, Craft JC. Drug interaction between clarithromycin and oral zidovudine in HIV 1 infected patients. Clin Pharmacol Ther 1993; 53: 163.

19 Parneix-Spake A, Bastuji-Garin S, Levy Y, Dubreuil Y, Lemaire M-L, Roujeau JC. Didanosine as a probable cause of StevensJohnson syndrome. Lancet 1992; 340: 857-8.

20 Buhles WC, Mastre BJ, Tinker AJ, Strand V, Koretz SH Ganciclovir treatment of life or sight threatening cytomegalovirus infection; experience in 314 immunocompromised patients. Rev ience in 314 immunocompromised pati
Infect Dis 1988; suppl 3: S495-S506.

21 Grossberg HS, Bonnem EM, Buhles WC. GM CSF with ganciclovir for the treatment of CMV retinitis in AIDS. N Engl f Med 1989; 320: 1560 .

22 Connolly GM, Gazzard MA, Hawkins DA Fixed drug eruption due to foscarnet. Genitourin Med 1990; 66: 97-8.
23 Gilquin J, Weiss K, Kazatchkine MD. Genital and oral ersions induced by foscarnet. Lance 1990; 335: 287.

24 Battegay M, Opravil M, Wuthrich B, Luthy R. Rash with amoxycillin-clavulanate therapy in HIV-infected patients. Lancet 1989; ii: 1100.

25 Williams I, Weller IVD, Malin A, Anderson J, Waters MFR. Thalidomide hypersensitivity in AIDS. Lancet 1991; 337: 436-7.

26 Wijnants H, Van den Ende J, Colebunders R. Multiple drug reactions in a patient with AIDS. Lancet 1989; ii: 1455 .

27 Ong ELC, Mandal BK. Multiple drug reactions in a patient with AIDS. Lancet 1989; ii: 976-7.

\section{Medical Anniversary \\ ANDREAS VESALIUS - 1 JANUARY 1514}

Andreas Vesalius (1514-64) was born in Brussels and was educated at Louvain and eventually in Paris where he studied medicine. He was a notable international lecturer, visiting Padua, Bologna and Pisa, where his public dissections earned him well-merited fame. He became personal physician to Emperor Charles V and Philip II of Spain. In June 1543 the first edition of his De humani corporis fabrica was printed in Basle. It is beautifully illustrated. A fine woodcut title page shows a bearded Vesalius dissecting a female at Padua in about 1540 before attentive onlookers. A naked model indicates the importance attached to surface markings and an articulated skeleton emphasises the importance of osteology. The presence of an ape and a dog underline the value of comparative anatomy.

He undertook a pilgrimage to Jerusalem and died on the island of Zante. - DG fames 